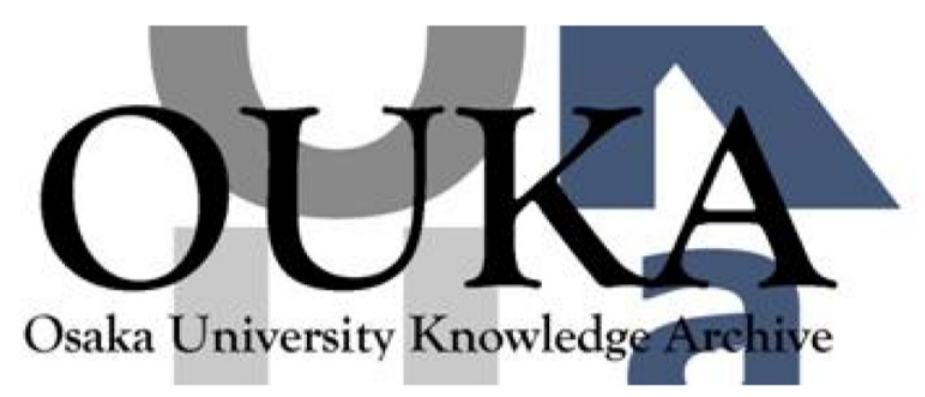

\begin{tabular}{|c|l|}
\hline Title & Composite models with chiral symmetry \\
\hline Author(s) & Višnjić, Vladimir; Higashijima, Kiyoshi \\
\hline Citation & $\begin{array}{l}\text { Physical review. Third series. D, Particles and } \\
\text { fields. 30(3) p. 655-p.659 }\end{array}$ \\
\hline Issue Date & $1984-08$ \\
\hline oaire:version & VoR \\
\hline URL & $\begin{array}{l}\text { https://hdl. handle. net/11094/3279 } \\
\text { Higashijima, Kiyoshi, Višnjić, Vladimir, } \\
\text { Physical review. Third series. D, Particles and } \\
\text { fields, 30, 3,655-659, 1984-08. "Copyright } \\
1984 \text { by the American Physical Society." }\end{array}$ \\
\hline rights \\
\hline Note & \begin{tabular}{l} 
\\
\hline
\end{tabular} \\
\hline
\end{tabular}

Osaka University Knowledge Archive : OUKA

https://ir. Library. osaka-u. ac. jp/

0saka University 


\title{
Composite models with chiral symmetry
}

\author{
Kiyoshi Higashijima and Vladimir Višnjić \\ Fermi National Accelerator Laboratory, P.O. Box 500, Batavia, Illinois 60510 \\ and School of Physics and Astronomy, University of Minnesota, Minneapolis, Minnesota 55455 \\ H. Suura \\ School of Physics and Astronomy, University of Minnesota, Minneapolis, Minnesota 55455
}

(Received 5 August 1983)

\begin{abstract}
Two model field theories involving scalar and fermion fields with contact interactions are analyzed. The models are solved in the large- $N$ limit. It is shown that chiral symmetry of the Lagrangian is realized in the spectrum. The anomalous magnetic moment of the composite fermions is shown to be $m_{F} / m_{B}{ }^{2}$, where $m_{F}$ and $m_{B}$ are the masses of the fermionic and bosonic preons, respectively. Finally a semirealistic model which incorporates the known gauge interactions is constructed.
\end{abstract}

\section{INTRODUCTION}

It is widely accepted that the main problem in constructing composite models for quarks and leptons consists in obtaining composite fermions whose mass is much smaller than the scale of binding. In the dynamical mechanisms proposed so far such fermions arise as massless "baryons" in a Yang-Mills theory with unbroken or partially broken chiral symmetry, ${ }^{1}$ as Goldstone fermions of dynamically broken supersymmetry, ${ }^{2}$ or as supersymmetric partners of Goldstone bosons of a spontaneously broken global symmetry. ${ }^{3}$ Although all these approaches have contributed to the development of new ideas, they have not produced a simple calculable model in which one could compute quantities such as masses of the bound states, their anomalous magnetic moments, etc.

In this paper we examine two model field theories involving scalar and fermion fields with contact interactions. These interactions are nonrenormalizable; therefore, we have to keep the cutoff as a physical parameter. We consider $N$-component scalar and fermion fields in the limit of large $N$ and solve the theory to the leading order in $1 / N$.

We find that in this limit the chiral symmetry of the Lagrangian is generally realized in the spectrum. The mass of the fermion-scalar bound state is proportional to the explicit chiral-symmetry-breaking terms such as, for example, the mass term for the fermionic field. In particular, the mass of the bound-state fermion does not depend on the mass of the scalar field.

We compute the electromagnetic couplings of the composite fermions with particular interest focused on their anomalous magnetic moments. They turn out to be of order $m_{F} / m_{B}{ }^{2}$, where $m_{F}$ and $m_{B}$ are the masses of the fermionic and bosonic constituents, respectively, thus confirming suggestions made earlier by several authors.

Finally, we show how to incorporate the gauge interactions in our model and construct a semirealistic model for quarks and leptons.

\section{THE FIRST MODEL}

In this section we discuss our simplest model, described by the Lagrangian

$$
\mathscr{L}_{1}=\bar{\psi}(i \partial-m) \psi+\phi^{\dagger}\left(-\square-\mu^{2}\right) \phi-\frac{g}{N} \phi \cdot \bar{\psi} \psi \cdot \phi^{\dagger},
$$

where $\phi \equiv\left(\phi_{1}, \phi_{2}, \ldots, \phi_{N}\right)$ and $\psi \equiv\left(\psi_{1}, \psi_{2}, \ldots, \psi_{N}\right)$ are $N$-component scalar and fermion fields, respectively. Note that the chiral symmetry is explicitly broken by the interaction and by the fermion mass term (if $m \neq 0$ ). The latter is included for the sake of generality and the zerofermion-mass limit of the theory is straightforward. The Lagrangian (2.1) is the simplest one describing interacting scalar and fermion fields. Since this theory is not renormalizable $\left[g\right.$ has dimension (mass) $\left.{ }^{-1}\right]$, we shall introduce an ultraviolet cutoff $\Lambda$ for the momentum integrations. Then our model is an analogy of the Nambu-JonaLasinio model $^{4}$ with fermionic bound states.

We now proceed to solve the theory in the leading order in $1 / N$ in the large- $N$ limit. The dominant graphs in this limit will be those containing the maximal number of scalar-fermion loops (Fig. 1). In order to sum up these diagrams, we transform the Lagrangian (2.1) to an equivalent Lagrangian of the Gaussian form with respect to $\psi$ and $\phi$ by adding the following term to it:

$$
-\frac{N}{g}\left[\bar{q}+\frac{g}{N} \bar{\psi} \cdot \phi \gamma_{5}\right]\left[q-\frac{g}{N} \gamma_{5} \phi^{\dagger} \cdot \psi\right] \text {. }
$$

The contribution of this term reduces to just a numerical



FIG. 1. The dominant contribution to the scalar-fermion bound state in the large- $N$ limit. 
constant in the path-integral formalism. The resulting equivalent Lagrangian is given by

$$
\begin{aligned}
\mathscr{L}= & \bar{\psi}(i \partial-m) \psi+\phi^{\dagger}\left(-\square-\mu^{2}\right) \phi-\frac{N}{g} \bar{q} q \\
& +\left(\bar{q} \gamma_{5} \psi \cdot \phi^{\dagger}-\bar{\psi} \cdot \phi \gamma_{5} q\right) .
\end{aligned}
$$

If we eliminate $\bar{q}$ and $q$ from this Lagrangian by using their equations of motion, we will recover the original Lagrangian, Eq. (2.1). As is seen from Eq. (2.2), $q$ represents a composite operator $\gamma_{5} \phi^{\dagger} \cdot \psi$, so that it describes a composite fermion if its propagator has a pole.

In order to find the mass of the bound state, we integrate out $\psi$ and $\phi$ fields in favor of $q$ and $\bar{q}$ and define the effective action for $q$ and $\bar{q}$ by

$$
\begin{aligned}
& \exp \left[i S_{\mathrm{eff}}(q, \bar{q})\right] \\
& \quad \equiv \int\left[d \phi_{i} d \phi_{i}^{\dagger} d \psi_{i} d \bar{\psi}_{i}\right] \exp \left(i \int \mathscr{L}(x) d x\right) .
\end{aligned}
$$

After the integrations over $\psi$ and $\phi$ are performed, we find

$$
\begin{aligned}
S_{\text {eff }} & =-\frac{N}{g} \int d x \bar{q} q+i N \operatorname{Tr} \ln \left[1-\frac{1}{\square+\mu^{2}} \bar{q} \gamma_{5} \frac{1}{i \partial-m} \gamma_{5} q\right) \\
& =S_{0}+S_{\text {int }},
\end{aligned}
$$

where $S_{0}$ denotes the bilinear term for $q$ and $S_{\text {int }}$ the rest:

$$
S_{0}=-\frac{N}{g} \int d x \bar{q} q-i N \operatorname{Tr}\left[\frac{1}{\square+\mu^{2}} \bar{q} \gamma_{5} \frac{1}{i \partial-m} \gamma_{5} q\right]
$$

and

$$
S_{\mathrm{int}}=-i N \sum_{n=2}^{\infty} \frac{1}{n} \operatorname{Tr}\left[\frac{1}{\square+\mu^{2}} \bar{q} \gamma_{5} \frac{1}{i \not \partial-m} \gamma_{5} q\right]^{n} .
$$

If we wish to include gauge fields, we have to replace $\square$ and $\partial$ with the appropriate covariant derivatives in Eqs. (2.6) and (2.7).

It is now easy to obtain the propagator of the field $q$ defined by

$$
S_{0}=\int d^{\dagger} x d^{4} y \bar{q}(x) i G^{-1}(x, y) q(y),
$$

namely,

$$
\begin{aligned}
i G^{-1}(p) & =-\frac{N}{g}+N \int \frac{d^{4} k}{(2 \pi)^{4} i} \frac{1}{\mu^{2}-(p-k)^{2}} \gamma_{5} \frac{1}{\not k-m} \gamma_{5} \\
& \equiv \not A\left(p^{2}\right)-B\left(p^{2}\right),
\end{aligned}
$$

where

$$
A\left(p^{2}\right)=\frac{N}{(4 \pi)^{2}} \frac{1}{2} \ln \frac{\Lambda^{2}}{\mu^{2}} \equiv Z^{-1}
$$

and

$$
B\left(p^{2}\right)=\frac{N}{g}+\frac{N m}{(4 \pi)^{2}} \ln \frac{\Lambda^{2}}{\mu^{2}}
$$

for $m<<\mu<\Lambda$.

In order to obtain the correct normalization, we introduce the renormalized composite-quark field and the renormalized coupling by

$$
q_{R} \equiv Z^{-1 / 2} q
$$

and

$$
g_{R} \equiv Z^{-1} g .
$$

Then the renormalized composite-quark propagator is given by

$$
i G_{R}^{-1}=Z i G^{-1}=\not p-M,
$$

where

$$
M=\frac{B\left(p^{2}\right)}{A}=2 m+\frac{1}{g_{R}} .
$$

As long as $m<<\mu<\Lambda$, the mass of the composite quark depends only logarithmically on the mass of the scalar preon and can be made arbitrarily small by having a light fermionic preon and the large cutoff $\Lambda$ (since $\left.g_{R} \propto \ln \Lambda / \mu^{2}\right)$.

At first glance this seems surprising-we obtain a light composite fermion by binding a light fermion and an arbitrarily heavy scalar. However, this result can be understood in terms of the approximate chiral symmetry of the effective action (2.5). Although our Lagrangian (2.1) does not have chiral symmetry as we pointed out earlier in this section, the effective action (2.5) has accidental chiral symmetry if we put $m=0$ and $1 / g=0$. Equation (2.15) gives the composite fermion mass in the large- $N$ limit as the sum of these chiral-symmetry-breaking terms. In the higher order of $1 / N$ expansion, the composite fermion will acquire additional mass by the interaction (2.7). Among others, the four-composite-fermion interaction of order $1 / N$ (Fig. 2) may lead to dynamical chiralsymmetry breaking. However, it is a well known property of the Nambu-Jona-Lasinio model ${ }^{4}$ that the chiral symmetry of the model is spontaneously broken only if the four-fermion coupling exceeds a critical value. Chiral symmetry is thus not spontaneously broken here because, by insisting that $N$ is large, we can make this coupling as small as we wish.

It should be emphasized that the chiral symmetry of the effective action (2.5) is an accidental symmetry and $1 / g=0$ is a singular limit. It is desirable to recast our analysis in terms of a theory which is completely chiral symmetric. We will postpone this analysis to Sec. IV and discuss the anomalous magnetic moment of the composite fermion.



FIG. 2. Diagrams which in the Nambu-Jona-Lasinio model lead to chiral-symmetry breaking if the four-fermion coupling exceeds a critical value. The heavy lines denote the compositefermion propagator. 


\section{ELECTROMAGNETIC INTERACTIONS AND THE ANOMALOUS MAGNETIC MOMENT}

Phenomenologically, the electromagnetic interactions, and, in particular, the anomalous magnetic moments, are very important tests of compositeness of quarks and leptons. ${ }^{5}$ We investigate here the electromagnetic properties of the composite fermions described in Sec. II.

Let $e_{F}$ and $e_{S}$ denote the charges of the $\psi$ and $\phi^{\dagger}$ fields, respectively. The electromagnetic interactions are then included by replacing the derivatives by the covariant derivatives,

$$
\begin{aligned}
& \partial \rightarrow \partial-i e_{F} A \equiv D, \\
& \square \rightarrow\left(\partial_{\mu}+i e_{S} A_{\mu}\right)^{2} \equiv D^{2}
\end{aligned}
$$

in the quadratic term of the action, Eq. (2.6), yielding

$$
\begin{aligned}
S^{(2)}\left(q, A_{\mu}\right)= & -i N \operatorname{Tr}\left[\frac{1}{D^{2}+\mu^{2}} \bar{q} \gamma_{5} \frac{1}{i \not D-m} \gamma_{5} q\right] \\
& -\frac{N}{g} \int \bar{q} q d^{4} x .
\end{aligned}
$$

We expand the propagators

$$
\frac{1}{D^{2}+\mu^{2}}=\frac{1}{\square+\mu^{2}}+\frac{1}{\square+\mu^{2}}\left(D^{2}-\square^{2}\right) \frac{1}{\square+\mu^{2}}+\cdots
$$



FIG. 3. The electromagnetic vertex of the composite fermion.

and

$$
\frac{1}{i \not D-m}=\frac{1}{i \partial-m}+\frac{1}{i \partial-m}(i \not D-i \partial) \frac{1}{i \partial-m}+\cdots
$$

and keeping only the linear terms in $A_{\mu}$,

$$
S^{(2)}\left(q, A_{\mu}\right)=S^{(2)}(q)+\Delta S^{(2)}(q),
$$

where

$$
\begin{aligned}
& \Delta S^{(2)}(q) \equiv N \int \frac{d^{4} k}{(2 \pi)^{4}} \int \frac{d^{4} p}{(2 \pi)^{4}} \bar{q}_{R}(-p-k) \\
& \times \Gamma^{\mu}(p, k) q_{R}(p) A_{\mu}(k) .
\end{aligned}
$$

The vertex function $\Gamma^{\mu}$, diagrammatically represented in Fig. 3, is

$Z \Gamma^{\mu}(p, k)=i e_{s} \int \frac{d^{4} p^{\prime}}{(2 \pi)^{4}} \frac{1}{\left(p^{\prime}+k\right)^{2}-\mu^{2}} \frac{\left(2 p^{\prime}+k\right)^{\mu}}{\mu^{2}-p^{\prime 2}} \gamma_{5} \frac{1}{p-p^{\prime}-m} \gamma_{5}+i e_{F} \int \frac{d^{4} p}{(2 \pi)^{4}} \frac{1}{\left(p-p^{\prime}\right)^{2}-\mu^{2}} \gamma_{5} \frac{1}{p^{\prime}+\not k-m} \gamma^{\mu} \frac{1}{p^{\prime}-m} \gamma_{5}$

In order to obtain the local effective Lagrangian, we expand $\Gamma^{\mu}(p, k)$ in powers of $k^{\mu}$

$$
\Gamma^{\mu}(p, k)=\Gamma_{0}^{\mu}(p)+k_{v} \Gamma^{\mu v}(p)+\cdots,
$$

$\Gamma_{0}^{\mu}$ and $\Gamma^{\mu v}$ are readily computed by expanding the propagators

$$
\frac{1}{\left(p^{\prime}+k\right)^{2} \mu^{2}}=\frac{1}{p^{\prime 2}-\mu^{2}}-\frac{1}{p^{\prime 2}-\mu^{2}} 2 p^{\prime} \cdot k \frac{1}{p^{\prime 2}-\mu^{2}}+\cdots
$$

and

$$
\frac{1}{p^{\prime}+\not k-m}=\frac{1}{p^{\prime}-m}-\frac{1}{p^{\prime}-m} k \frac{1}{p^{\prime}-m}+\cdots .
$$

For $Z \Gamma_{0}^{\mu}$ we obtain

$$
\begin{aligned}
Z \Gamma_{0}^{\mu}(p) & =-i e_{s} \int \frac{d^{4} p^{\prime}}{(2 \pi)^{4}} \frac{2 p^{\prime \mu}}{\left(p^{\prime 2}-\mu^{2}\right)^{2}} \gamma_{5} \frac{1}{p-p^{\prime}-m} \gamma_{5}+i e_{F} \int \frac{d^{4} p^{\prime}}{(2 \pi)^{4}} \frac{1}{\left(p-p^{\prime}\right)^{2}-\mu^{2}} \gamma_{5} \frac{1}{p^{\prime}-m} \gamma^{\mu} \frac{1}{p^{\prime}-m} \gamma_{5} \\
& =\left(e_{s}+e_{F}\right) \gamma^{\mu} \frac{1}{2(4 \pi)^{2}} \ln \frac{\Lambda^{2}}{\mu^{2}}
\end{aligned}
$$

and for the renormalized vertex function

$$
\Gamma_{0}^{\mu}(p)=\left(e_{s}+e_{F}\right) \gamma^{\mu} \equiv e \gamma^{\mu} .
$$

As expected, the bound state couples to the photon as a fermion, with the strength determined by the total charge.

Our main goal in this section is to calculate the anomalous magnetic moment of the bound state, which is obtained as the antisymmetric part of $Z \Gamma^{\mu \nu}(p)$ :

$$
Z\left(\Gamma^{\mu v}-\Gamma^{v \mu}\right)=\frac{2 i e}{(4 \pi)^{2}} m \sigma^{\mu v} \frac{1}{\mu^{2}} \ln \frac{\mu^{2}}{m^{2}} .
$$

The effective Lagrangian is

$$
\mathscr{L}_{\text {eff }}=N \frac{e m}{\mu^{2}} \frac{\ln \left(\mu^{2} / m^{2}\right)}{\ln \left(\Lambda^{2} / \mu^{2}\right)} \bar{q}_{R} \sigma^{\mu v} q_{R} F_{\mu \nu},
$$

where $q_{R} \equiv Z^{-1 / 2} q$, with $q=(g / N) \gamma_{5} \phi^{\dagger} \cdot \psi$, is the renormalized composite fermion field. The anomalous magnetic moment is proportional to the mass of the fermionic constituent and inversely proportional to the scalar mass. Although this was suggested by several people, ${ }^{6}$ we find it reassuring that in our model that can actually be confirmed by explicit calculation. 
One might attempt to construct a realistic composite model for quarks and leptons based on the mode proposed here. However, there appears to be no simple way of incorporating the gauge theory of weak interactions. The problem is that the interaction term in the Lagrangian (2.1), not being chirally invariant, breaks $\mathrm{SU}(2)$ gauge symmetry.

\section{THE SECOND MODEL}

The difficulties in incorporating the weak interactions in the model described in the preceding sections are related to the fact that the interaction is not chirally invariant. For that reason we study here a similar model, but with a contact interaction which is chirally invariant. The Lagrangian of the model is

$\mathscr{L}_{2}=\bar{\psi}(i \partial-m) \psi+\phi^{\dagger}\left(-\square-\mu^{2}\right) \phi-\frac{g}{N}(\bar{\psi} \cdot \phi) i \partial /\left(\psi \cdot \phi^{\dagger}\right)$,

where the notation is the same as in Sec. III, except that the coupling constant $g$ now has dimension (mass) ${ }^{-2}$. Chiral symmetry is now broken only by the possible fermion mass term, and if it is not spontaneously broken we might expect the mass of the composite fermion to be proportional to $m$.

In order to solve the theory (4.1) in the large- $N$ limit we add the following term $\mathscr{L}_{2}$ :

$$
\frac{N}{g}\left[\bar{q}-\frac{g}{N} \phi \cdot \bar{\psi} \gamma_{5} i \partial\right](i \partial)^{-1}\left[q+\frac{g}{N} i \partial \gamma_{5} \psi \cdot \phi^{\dagger}\right],
$$

leading to the following equivalent Lagrangian:

$$
\begin{aligned}
\mathscr{L}= & \bar{\psi}(i \partial-m) \psi+\phi^{\dagger}\left(-\square-\mu^{2}\right) \phi \\
& +\left(\bar{q} \gamma_{5} \psi \cdot \phi^{\dagger}-\bar{\psi} \cdot \phi \gamma_{5} q\right)+\frac{N}{g} \bar{q}(i \partial)^{-1} q .
\end{aligned}
$$

This Lagrangian, except for the mass term for $\psi$, is invariant under chiral rotation,

$$
\begin{aligned}
& \psi \rightarrow e^{i \gamma_{5} \theta} \psi, \phi \rightarrow \phi, \\
& q \rightarrow e^{-i \gamma_{5} \theta} q .
\end{aligned}
$$

Since the composite fermion $q$ transforms nontrivially under this chiral transformation, $q$ remains massless in the chiral limit ( $m=0$ ) if the chiral symmetry is realized in the Wigner phase. [Another chiral-symmetric extension of Eq. (2.1) would be

$$
-\frac{g}{N}\left(\phi \cdot \bar{\psi}_{R} \psi_{L} \cdot \phi+\phi^{\dagger} \cdot \bar{\psi}_{L} \psi_{R} \cdot \phi^{\dagger}\right)
$$

which is invariant under the chiral symmetry $\psi \rightarrow e^{i \theta \gamma_{5}} \psi, \phi \rightarrow e^{i \theta} \phi$. In this case, however, the composite field

$$
q \sim \frac{g}{N}\left(\psi_{R} \cdot \phi+\psi_{L} \cdot \phi^{\dagger}\right)
$$

transforms trivially under the chiral symmetry; thereby $q$ acquires mass even in the chiral-symmetry limit $(m=0)$.]

The calculations are done analogously to those in Sec.
III and the result for the propagator of the composite field $q$ can again be written as

$$
i G^{-1}(p)=\not p A\left(p^{2}\right)-B\left(p^{2}\right),
$$

where now

$$
A\left(p^{2}\right)=\frac{N}{2(4 \pi)^{2}} \ln \frac{\Lambda^{2}}{\mu^{2}}+\frac{N}{g} \frac{1}{p^{2}}
$$

and

$$
B\left(p^{2}\right)=\frac{N m}{(4 \pi)^{2}} \ln \frac{\Lambda^{2}}{\mu^{2}} .
$$

When $\Lambda^{2} \gg \mu^{2}, m^{2}, p^{2}$, the bound-state propagator becomes

$$
i G^{-1}(p)=Z^{-1}(p-2 m) .
$$

Thus, the mass of the bound state is twice the mass of the fermionic preon and independent of the mass of the scalar preon. Chiral symmetry of the Lagrangian is realized in the spectrum and in the presence of explicit breaking (i.e., $m \neq 0$ ) the bound state acquires mass directly proportional to the magnitude of the symmetry-breaking term.

It should be emphasized that the effective action of the second model, obtained from Eq. (2.5) by replacing $-\bar{q} q$ with $\bar{q}(i \partial)^{-1} q$, is completely chiral symmetric when $m=0$, reflecting the chiral symmetry of the Lagrangian (4.1). It is interesting to note that the one-loop contribution (the second term) in Eq. (2.5) is chiral symmetric when $m=0$, in spite of the symmetry-breaking interaction. The origin of this symmetry for chiral-noninvariant interaction is not clear to the authors at the moment.

\section{A MODEL OF QUARKS AND LEPTONS}

Since our model is a nonconfining one, the preon $\psi$ has to be an observed particle as well as the composite fermion $q$. It is tempting to identify $\psi$ and $q$ with leptons and quarks. The first model of a similar kind, in which $\psi$ and $q$ are identified as leptons and baryons, was called the Nagoya model. ${ }^{7}$

In this section we present a semirealistic model based on this idea. In order to incorporate the strong, weak, and electromagnetic interactions we have to use the chiralsymmetric Lagrangian (4.1), since the simplest Lagrangian (2.1) breaks gauge invariance. For simplicity we discuss the model of one generation of leptons and quarks.

We assume the standard assignment for leptons, namely,

$$
\begin{aligned}
& \psi_{L}=\left[\begin{array}{l}
v \\
e
\end{array}\right)_{L}, \quad I=\frac{1}{2}, Y=-1, \\
& \psi_{R}=v_{R} \text { and } e_{R}, I=0, Y=0 \text { and } I=0, Y=-2 .
\end{aligned}
$$

The right-handed neutrino, of course, does not couple to the gauge bosons. The scalars appear in a cooler triplet $\phi$, with $I=0$ and $Y=-\frac{2}{3}$ and a color singlet

$$
H=\left(\begin{array}{c}
H^{+} \\
H^{0}
\end{array}\right)
$$

with $I=\frac{1}{2}$ and $Y=1$.

Our model Lagrangian for these fields is 


$$
\begin{aligned}
\mathscr{L}= & \bar{\psi}_{L} i \not \psi_{L}+\bar{v}_{R} i \not v_{R}+\bar{e}_{R} i \emptyset e_{R}+\phi^{\dagger}\left(-D^{2}-\mu^{2}\right) \phi-f\left(\bar{\psi}_{L} H e_{R}+\text { H.c. }\right) \\
& -\frac{g}{N}\left[\left(\bar{\psi}_{L} \phi\right) i \not\left(\psi_{L} \phi^{\dagger}\right)+\left(\bar{e}_{R} \phi\right) i \not\left(e_{R} \phi^{\dagger}\right)+\left(\bar{v}_{R} \phi\right) i \not\left(v_{R} \phi^{\dagger}\right)\right]
\end{aligned}
$$

where

$$
D_{\mu} \psi=\left(\partial_{\mu}-i g I_{\alpha} W_{\mu}^{\alpha}-i g^{\prime} \frac{1}{2} \gamma B_{\mu}\right) \psi
$$

with $I_{\alpha}=\frac{1}{2} \tau_{\alpha}$ for $I=\frac{1}{2}$ and zero for $I=0$, and

$$
D_{\mu} \phi=\left(\partial_{\mu}-i g_{s} \frac{1}{2} \lambda_{\alpha} A_{\mu}^{\alpha}-i g^{\prime} \frac{1}{2} Y B_{\mu}\right) \phi \text {. }
$$

$A_{\mu}^{\alpha}, W_{\mu}^{\alpha}$, and $B_{\mu}$ are the gauge fields of $\mathrm{SU}(3)_{C}, \mathrm{SU}(2)_{L}$, and $\mathrm{U}(1)$ interactions.

As in the previous sections we add the following terms to the Lagrangian:

$$
\begin{aligned}
& \frac{N}{g}\left[\bar{q}_{R}^{c}-\frac{g}{N} \phi \bar{\psi}_{L} i \not D\right](i \not D)^{-1}\left[q_{R}^{c}-\frac{g}{N} i \not D \psi_{L} \phi^{\dagger}\right]+\frac{N}{g}\left[\bar{u}_{L}^{c}+\frac{g}{N} \phi \bar{e}_{R} i \not D(i \not)^{-1}\left[u_{L}^{c}+\frac{g}{N} i \not D e_{R} \phi^{\dagger}\right]\right. \\
& \quad+\frac{N}{g}\left[\bar{d}_{L}^{c}+\frac{g}{N} \phi \bar{v}_{R} i \not D\right](i D)^{-1}\left[d_{L}^{c}+\frac{g}{N} i \not v_{R} \phi^{\dagger}\right],
\end{aligned}
$$

where the superscript $c$ indicates the charge-conjugate fields, and

$$
q_{R}^{c} \equiv \sigma_{2}\left(\begin{array}{l}
u_{R}^{c} \\
d_{R}^{c}
\end{array}\right)=i\left(\begin{array}{c}
-d_{R}^{c} \\
u_{R}^{c}
\end{array}\right) .
$$

With that we obtain the linearized Lagrangian

$$
\begin{aligned}
\mathscr{L}= & \bar{\psi}_{L} i \not \psi_{L}+\bar{v}_{R} i \not v_{R}+\bar{e}_{R} i \not e_{R}+\phi^{\dagger}\left(-D^{2}-\mu^{2}\right) \phi-f\left(\bar{\psi}_{L} H e_{R}+\text { H.c. }\right)+\frac{N}{g}\left[\bar{q}_{R}^{c}(i \not)^{-1} q_{R}^{c}+\bar{u}_{L}^{c}(i \not D)^{-1} u_{L}^{c}+\bar{d}_{L}^{c}(i \not)-1 d_{L}^{c}\right] \\
& -\left(\bar{q}_{R}^{c} \psi_{L} \phi^{\dagger}+\phi \bar{\psi}_{L} q_{R}^{c}-\bar{u}_{L}^{c} e_{R} \phi^{\dagger}-\phi \bar{e}_{R} u_{L}^{c}-d_{L}^{c} v_{R} \phi^{\dagger}-\phi \bar{v}_{R} d_{L}^{c}\right) .
\end{aligned}
$$

Variations with respect to $q_{R}^{c}, u_{L}^{c}$, and $d_{L}^{c}$ give

$$
\begin{aligned}
& q_{R}^{c}=\frac{g}{N} i \not\left(\psi_{L} \phi^{\dagger}\right), \\
& u_{L}^{c}=-\frac{g}{N} i \emptyset\left(e_{R} \phi^{\dagger}\right), \\
& d_{L}^{c}=-\frac{g}{N} i \not\left(v_{R} \phi^{\dagger}\right) .
\end{aligned}
$$

The reason why we have to use the charge-conjugate fields is that, e.g., $\emptyset\left(\psi_{L} \phi^{\dagger}\right)$ is a right-handed field with the weak isospin $\frac{1}{2}$, and cannot be assigned to either $q_{L}$ or $q_{R}$. Hence, it is assigned to $q_{R}^{c}$ which has the same quantum numbers.

By eliminating the $\phi$ field we obtain the Lagrangian of the standard model $^{8}$ for quarks and leptons as an effective Lagrangian of our composite model, Eq. (5.1). In the framework of our model this essentially completes the program which the realistic model for composite quarks and leptons will eventually have to fulfill: starting from the fundamental Lagrangian, obtain the mass spectrum of the composite fermion, and derive the standard model as an effective low-energy theory.

The main purpose of this paper was to present a sufficiently simple model in which this program can be carried through explicitly. From the point of view of phenomenology, the main criticism of the model described here is that it does not appear to offer a clue as to the generation problem. One could, of course, have preons appearing in generations, but to our mind that goes against the main philosophy of having composite quarks and leptons. The contact interactions, Eq. (4.1), is certainly too simple to produce the generation structure dynamically.
${ }^{1} \mathrm{G}$. 't Hooft, in Recent Developments in Gauge Theories edited by G. 't Hooft et al. (Plenum, New York, 1980). For an exhaustive list of references, see L. Lyons, Prog. Part. Nucl. Phys. 10, 227 (1983).

${ }^{2}$ W. A. Bardeen and V. Višnjić, Nucl. Phys. B 194, 422 (1982).

${ }^{3}$ W. Buchmüller, S. T. Love, R. D. Peccei, and T. Yanagida, Phys. Lett. 115B, 233 (1982); R. Barbieri, A. Masiero, and G. Veneziano, ibid. 128B, 179 (1983); A. Buras and W. Slominski, Nucl. Phys. B223, 157 (1983).

${ }^{4}$ Y. Nambu and G. Jona-Lasinio, Phys. Rev. 122, 345 (1961).

${ }^{5}$ E. Eichten, K. Lane, and M. Peskin, Phys. Rev. Lett. 50, 811
(1983).

${ }^{6}$ V. Višnjić, Phys. Lett. 95B, 47 (1980); S. J. Brodsky and S. D. Drell, Phys. Rev. D 22, 2236 (1980).

${ }^{7}$ Z. Maki, M. Nakagawa, Y. Ohnuki, and S. Sakata, Prog. Theor. Phys. 23, 1274 (1960); Z. Maki, M. Nakagawa, and S. Sakata, ibid. 28, 870 (1962).

${ }^{8}$ S. Weinberg, Phys. Rev. Lett. 19, 1264 (1967); A. Salam, in Elementary Particle Theory: Relativistic Groups and Analyticity (Nobel Symposium No. 8), edited by N. Svartholm (Almqvist and Wiksell, Stockholm, 1968), p. 367. 